\title{
Determination of energy-band offsets between GaN and AIN using excitonic luminescence transition in AIGaN alloys
}

\author{
A. N. Westmeyer, S. Mahajan, and K. K. Bajaj ${ }^{\text {a) }}$ \\ Department of Chemical and Materials Engineering, Arizona State University, Tempe, Arizona 85287 \\ J. Y. Lin and H. X. Jiang \\ Department of Physics, Kansas State University, Manhattan, Kansas 66506 \\ D. D. Koleske \\ Sandia National Laboratories, Albuquerque, New Mexico 87185
}

R. T. Senger

Department of Physics, Bilkent University, 06800 Ankara, Turkey

(Received 5 April 2005; accepted 18 November 2005; published online 9 January 2006)

\begin{abstract}
We report the determination of the energy-band offsets between $\mathrm{GaN}$ and $\mathrm{AlN}$ using the linewidth (full width at half maximum) of an extremely sharp excitonic luminescence transition in $\mathrm{Al}_{x} \mathrm{Ga}_{1-x} \mathrm{~N}$ alloy with $x=0.18$ at $10 \mathrm{~K}$. Our sample was grown on $C$-plane sapphire substrate by metal-organic chemical-vapor deposition at $1050{ }^{\circ} \mathrm{C}$. The observed value of the excitonic linewidth of $17 \mathrm{meV}$ is the smallest ever reported in literature. On subtracting a typical value of the excitonic linewidth in high-quality $\mathrm{GaN}$, namely, $4.0 \mathrm{meV}$, we obtain a value of $13.0 \mathrm{meV}$, which we attribute to compositional disorder. This value is considerably smaller than that calculated using a delocalized exciton model [S. M. Lee and K. K. Bajaj, J. Appl. Phys. 73, 1788 (1993)]. The excitons are known to be strongly localized by defects and/or the potential fluctuations in this alloy system. We have simulated this localization assuming that the hole, being much more massive than the electron, is completely immobile, i.e., the hole mass is treated as infinite. Assuming that the excitonic line broadening is caused entirely by the potential fluctuations experienced by the conduction electron, the value of the conduction-band offset between $\mathrm{GaN}$ and $\mathrm{AlN}$ is determined to be about $57 \%$ of the total-band-gap discontinuity. Using our model we have calculated the variation of the excitonic linewidth as a function of $\mathrm{Al}$ composition in our samples with higher $\mathrm{Al}$ content larger than $18 \%$ and have compared it with the experimental data. We also compare our value of the conduction-band offset with those recently proposed by several other groups using different techniques. () 2006 American Institute of Physics. [DOI: 10.1063/1.2158492]
\end{abstract}

\section{INTRODUCTION}

During the past few years, an enormous amount of effort of the semiconductor research community has been devoted to the study of the structural, electronic, and optical properties of III-V nitrides, their alloys, and their heterostructures. The motivation for this effort has been provided by applications in a variety of electronic and optoelectronic devices, such as high-power field-effect transistors, light-emitting diodes, and laser diodes operating in the visible to ultraviolet range of the electromagnetic spectrum. These devices have been fabricated using metal-organic chemical-vapor deposition (MOCVD) and molecular-beam epitaxy (MBE) growth techniques, and their performance characteristics have been studied in considerable detail. A number of these devices are now commercially available. In addition, a large number of experimental and theoretical investigations have been carried out to gain a better understanding of the fundamental properties of these materials. Although we have obtained a wealth of information during the past few years, a full understand-

\footnotetext{
${ }^{a)}$ Permanent address: Department of Physics, Emory University, Atlanta, GA 30322; electronic mail: phskkb@physics.emory.edu
}

ing of the various mechanisms involved in the operation of these devices and of the fundamental properties of these material systems is still lacking.

Several groups have investigated the optical properties of an AlGaN alloy system using a variety of characterization techniques such as absorption, photoluminescence excitation (PLE), cathodoluminescence, and photoluminescence (PL) spectroscopies during the past few years. In particular, the variation of the linewidth, defined as the full width at half maximum $(\sigma)$ of an excitonic transition at low temperatures, as a function of alloy composition in the AlGaN alloy system has been studied by several groups ${ }^{1-4}$ using PL spectroscopy. It is well known that such studies can provide useful information about the quality of the alloys. ${ }^{5}$ In their pioneering work, Steude et al. ${ }^{1}$ studied the optical properties of coherently strained $\mathrm{Al}_{x} \mathrm{Ga}_{1-x} \mathrm{~N}$ alloys $(0 \leqslant x \leqslant 0.22)$ grown on (0001)-oriented sapphire by MOCVD using a variety of characterization techniques such as absorption, transmission, reflection, cathodoluminescence, and PL at low temperatures. In particular, they have measured the variation of $\sigma$ as a function of $\mathrm{Al}$ concentration $(x)$ at $4 \mathrm{~K}$ and found, as expected, that it increases as a function of $x$.

Following this work, Coli et $a l^{2,3}$ have reported a study 
of the variation of $\sigma$ as a function of the $\mathrm{Al}$ concentration in $\mathrm{Al}_{x} \mathrm{Ga}_{1-x} \mathrm{~N}$ alloys using PL spectroscopy at $10 \mathrm{~K}$. In their studies the value of $x$ was varied from 0 to 0.7 . Their samples were grown by low-pressure MOCVD on sapphire (0001) substrates. They found that the values of $\sigma$ increased as a function of $x$, as expected, but are somewhat smaller than those measured by Steude et al. ${ }^{1}$ In addition, the measured values of $\sigma$ are close to those calculated by Lee and Bajaj, ${ }^{6}$ who assumed completely random alloys and delocalized excitons. Both Steude et al. ${ }^{1}$ and Coli et al., ${ }^{2,3}$ however, did not provide any information on the structural characteristics of their samples. Pendlebury et al. ${ }^{4}$ have also measured the variation of $\sigma$ with $\mathrm{Al}$ concentration in $\mathrm{Al}_{x} \mathrm{Ga}_{1-x} \mathrm{~N}$ using PL spectroscopy at $4 \mathrm{~K}$. Their samples were grown by MOCVD on $C$-plane sapphire substrates and the Al concentration was varied from 0 to 0.5 . Their values of $\sigma$ are larger than those reported by Coli et al. ${ }^{2,3}$ and are comparable to those measured by Steude et al. ${ }^{1}$ They also performed PLE measurements and measured the variation of Stokes's shift, i.e., the difference in energy between the peak positions in PLE and PL and find that it increases as a function of $\mathrm{Al}$ concentration, thus suggesting a strong localization of excitons in their samples. Recently, Cho et al. ${ }^{7}$ have investigated the optical properties of $\mathrm{Al}_{x} \mathrm{Ga}_{1-x} \mathrm{~N}$ epilayers using a variety of characterization techniques such as PL, PLE, and timeresolved PLE. Their samples were grown by MOCVD on (0001)-oriented sapphire substrates and the value of the $\mathrm{Al}$ concentration was varied from 0 to 0.6 . They measured the variation of the energy of the PL peak position as a function of temperature and observed a well-known "S-shaped" behavior (decrease-increase-decrease). In addition, the excitonic linewidth follows an "inverted S-shaped" behavior (increase-decrease-increase) as a function of temperature. These are well known signatures of emission due to localized excitons. Cho et al. ${ }^{7}$ also did not provide any information concerning the structural properties of their epilayers.

In this paper, we present results of structural and lowtemperature PL measurements on an $\mathrm{Al}_{x} \mathrm{Ga}_{1-x} \mathrm{~N}$ sample with Al concentration $x=0.18$. We use this information to calculate the values of the band offsets between GaN and AlN using a model where the holes are completely localized. We have used this model to calculate the variation of the excitonic linewidth as a function of $\mathrm{Al}$ concentration in our samples which have $\mathrm{Al}$ content larger than $18 \%$ and have compared it with the experimental data.

\section{EXPERIMENTAL DETAILS}

Our sample with $18 \% \mathrm{Al}$ concentration was grown by MOCVD and consisted of a thin $(10 \mathrm{~nm}) \mathrm{GaN}$ nucleation layer grown on $C$-plane sapphire at $550{ }^{\circ} \mathrm{C}$, a $1 \mu \mathrm{m}$-thick $\mathrm{GaN}$ layer grown on the nucleation layer at $1050{ }^{\circ} \mathrm{C}$, and a 2000- $\AA$-thick $\mathrm{Al}_{x} \mathrm{Ga}_{1-x} \mathrm{~N}$ layer also grown at $1050{ }^{\circ} \mathrm{C}$. No cap layer was used in this sample. Several analytical and structural measurements were performed using Rutherford backscattering (RBS), x-ray diffraction (XRD), and transmission electron microscopy (TEM). All PL measurements were performed at $10 \mathrm{~K}$ using a single-photon counting detection system together with a microchannel-plate photomultiplier

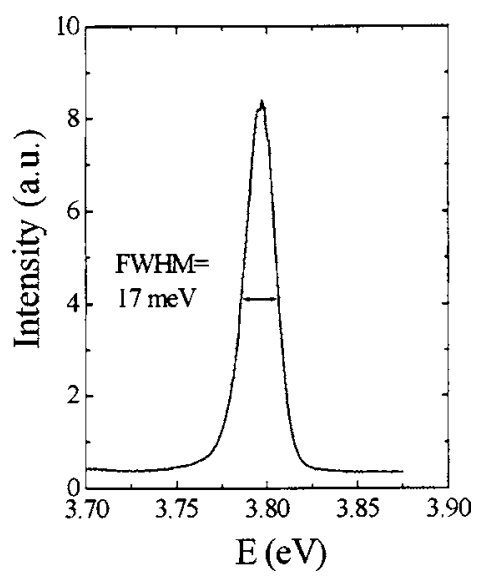

FIG. 1. PL spectrum of the $\mathrm{Al}_{0.18} \mathrm{Ga}_{0.82} \mathrm{~N}$ sample at $10 \mathrm{~K}$.

tube, with the detection capability ranging from 185 to $800 \mathrm{~nm}$ and an overall resolution of $0.2 \mathrm{meV}$. The wavelength of the exciting radiation was $292 \mathrm{~nm}$, and the PL signals were collected in reflecting mode at an incident angle of about $30^{\circ}$.

\section{RESULTS AND DISCUSSION}

The value of the Al concentration $x$ in our sample was determined using Rutherford backscattering and x-raydiffraction techniques. The latter measurement did not show any atomic ordering. This result was confirmed by examining the $[10 \overline{1} 0]$ zone-axis diffraction pattern (ZADP), where the [0001] spot cannot arise due to double diffraction. Our TEM results indicate the presence of composition modulations along the [0001] direction with a period of $(1.1 \pm 0.7) \mathrm{nm}$. In Fig. 1, we display the PL spectrum of $\mathrm{Al}_{x} \mathrm{Ga}_{1-x} \mathrm{~N}$ for $x=0.18$ at $10 \mathrm{~K}$.

During the past 20 years a number of groups ${ }^{5}$ have reported calculations of $\sigma$ as a function of alloy composition in completely disordered semiconductor alloys. The results of these calculations, as well as their comparison with the experimental data in a number of III-V and II-VI alloys, have been reviewed recently. ${ }^{5}$ We will compare our measured value of $\sigma$ with those calculated using the model of Lee and Bajaj. ${ }^{6}$ Using a quantum statistical approach, they obtained the following expression for $\sigma$ :

$$
\sigma=0.41 \sigma_{0}
$$

where

$$
\sigma_{0}=\frac{d E_{g}(x)}{d x}\left[\frac{8 \ln (2) x(1-x) V_{c}}{(4 \pi / 3) R_{\mathrm{ex}}^{3}}\right]^{1 / 2} .
$$

Here $V_{c}$ is the volume of the primitive cell, $d E_{g}(x) / d x$ describes the variation of the energy of the band gap with alloy composition, $R_{\mathrm{ex}}=\varepsilon_{0} \hbar^{2} / \mu e^{2}$ is the Bohr radius, $\mu=\left(1 / m_{e}\right.$ $\left.+1 / m_{h}\right)^{-1}$ is the reduced mass, $m_{e}$ and $m_{h}$ are the electron and the hole mass, respectively, and $\varepsilon_{0}$ is the static dielectric constant. The values of $m_{e}$ and $\varepsilon_{0}$ in $\mathrm{Al}_{x} \mathrm{Ga}_{1-x} \mathrm{~N}$ used in our calculations are obtained from those of GaN and AlN (Ref. 2) by linear interpolation, namely, 


$$
\frac{m_{e}(x)}{m_{0}}=0.22(1-x)+0.33 x
$$

and

$$
\varepsilon_{0}(x)=9.7(1-x)+6.3 x .
$$

The values of the hole masses associated with the topmost valence bands in GaN and AlN are not reliably known. A number of attempts, however, to determine the value of the average hole mass of the topmost valence band in $\mathrm{GaN}$ have been made and are reviewed by Vurgaftman et al. ${ }^{8}$ and by Vurgaftman and Meyer. ${ }^{9}$ These values range from $0.3 m_{0}$ to $2.2 m_{0}$. They suggest a value of $1.0 m_{0}$, based on the analysis of the available experimental data. There is practically no experimental information available about the hole mass of the topmost valence band of AlN. In addition, in AlN the crystal-field splitting is negative and thus the topmost valence band has a different symmetry than that in GaN. In view of these uncertainties, we use a value of $1.0 m_{0}$ for the hole mass in our sample which has a low (18\%) Al concentration. As the value of the reduced mass is primarily determined by the mass of lighter particle, namely, the electron, this assumption about the hole mass should not introduce any significant error. The volume is $V_{c}(x)=a_{0}^{3}(x) / \sqrt{2}$ where $a_{0}(x)=3.16(1-x)+3.112 x \AA$ is the lattice constant in the basal plane. The variation of the energy of the band gap $E_{g}(x)$ with the $\mathrm{Al}$ concentration is given as

$$
E_{g}(x)=(1-x) E_{g}(\mathrm{GaN})+x E_{g}(\mathrm{AlN})-b(1-x) x,
$$

where $b$ is the bowing parameter and the values of the energy gaps at $10 \mathrm{~K}$ are $E_{g}(\mathrm{GaN})==3.504 \mathrm{eV}$ and $E_{g}(\mathrm{AlN})$ $=6.285 \mathrm{eV}$. The value of the bowing parameter $b$ we used in our calculation is taken to be $0.6 \mathrm{eV}$. $^{2}$

As shown in Fig. 1, the line shape of the excitonic transition is almost Gaussian with the value of $\sigma$ of $17.0 \mathrm{meV}$. In order to consider only the effect of the compositional alloy disorder on $\sigma$ we subtract from $17.0 \mathrm{meV}$ the constant value of the inhomogeneous broadening $\sigma(\mathrm{GaN})$, namely, $4.0 \mathrm{meV}$, a typical value found in many high-quality samples grown on sapphire substrates in our MOCVD reactor. The value of $\sigma$ of $13.0 \mathrm{meV}$ thus obtained is the lowest value of the excitonic linewidth ever reported in $\mathrm{Al}_{x} \mathrm{Ga}_{1-x} \mathrm{~N}$ with $x$ $=0.18$. The value of $\sigma$ calculated for this sample using the formalism of Lee and Bajaj ${ }^{6}$ is $16.5 \mathrm{meV}$, larger than $13.0 \mathrm{meV}$. The observed value of the linewidth in our sample is also considerably smaller than the best reported value in literature. ${ }^{2}$ Any deviation from the perfectly random distribution of cations in our sample will only enhance the value of $\sigma$. It should be pointed out that the measured value of $\sigma$ in our sample also contains contributions from other mechanisms such as those due to the presence of random electric fields which arise from the significant additional incorporation of ionized impurities due to the presence of $\mathrm{Al}$.

As mentioned earlier, the emission in AlGaN alloys at low temperatures is primarily due to highly localized excitons. Such is also the case in a number of other semiconductor alloys such as $\mathrm{InGaP}^{10}{ }^{10} \mathrm{GaAsN},{ }^{11} \mathrm{InGaAsN}^{12}$ and GaAsSbN. ${ }^{13}$ The microscopic nature of excitonic localization by potential fluctuations and/or defects in alloys is not known. To simulate the exciton localization, Zeman et al. ${ }^{10}$ assumed complete localization of the hole (namely, the hole mass was treated as infinite) in InGaP layers lattice matched to GaAs and explained their data on the variation of the diamagnetic shift of the localized excitons with magnetic field at low temperatures. A similar assumption has also been made recently by Senger et al. ${ }^{13,14}$ to explain their PL data on the variation of the diamagnetic shift of the localized excitons with magnetic field in GaAsSb/GaAs and GaAsSbN/GaAs quantum well structures, respectively, at low temperatures. It seems that the assumption that the holes are completely localized best describes the situation of excitons localized by potential fluctuations and/or defects in the above-mentioned alloys. We believe that such is also the case in AlGaN alloys. If we assume that the holes due to their much larger masses are completely localized, namely, we treat the hole mass as infinite then only the electrons experience the fluctuating potential of the conduction band. The hole clearly does not experience any potential fluctuations. Substituting this value of the hole mass and replacing $d E_{g} / d x$ by $d E_{c} / d x$ we find that for $\sigma=13.0 \mathrm{meV}$, the value of the conduction-band discontinuity is such that $d E_{c} / d x$ $=0.57\left(d E_{g} / d x\right)$.

Recently, Foxon et al. ${ }^{15}$ have studied the behavior of the yellow and blue emissions in $\mathrm{GaN}$ and $\mathrm{Al}_{0.2} \mathrm{Ga}_{0.8} \mathrm{~N}$ epilayers. The yellow emission is assumed to be due to the recombination of a shallow donor with a deep acceptor due to $\mathrm{Ga}$ vacancy, and the blue emission is due to the recombination of a deep donor (presumably As antisite) with a shallow acceptor. Using the fact that the positions of deep levels in semiconductors against common vacuum reference level do not change with alloy composition, they determined the value of the conduction-band offset of 0.55 of the band-gap discontinuity. Helman et al. ${ }^{16}$ have studied the intersubband transitions due to conduction electrons in GaN/AlN quantum wells and have found that a conduction-band offset of 0.64 best explains their data. These two values of the conductionband offsets are quite close to the value we calculated.

Recently, Vurgaftman and Meyer ${ }^{9}$ have briefly reviewed the results of various calculations of the energy-band offsets between GaN and AlN. Satta et al. ${ }^{17}$ have pointed out that the energy-band offsets for GaN/AlN and AlN/GaN are different. From a detailed treatment of the strain-induced asymmetry at the (0001) polar heterojunctions Bernardini and Fiorentini ${ }^{18}$ have obtained $2.55 \mathrm{eV}(93 \%)$ and $1.9 \mathrm{eV}(69 \%)$ for the values of the conduction-band offsets for AlN/GaN and GaN/AlN interfaces, respectively. Vurgaftman and Meyer ${ }^{9}$ have also briefly reviewed the results of various measurements of the conduction-band offsets between $\mathrm{GaN}$ and AlN and found that these values range from $95 \%$ to $50 \%$ of the total-band discontinuity. Such an enormous disparity among the values of the conduction-band offset is ascribed to differences in effects of strain inhomogeneity, defects, and stoichiometry among the samples used by various groups. The consensus is, however, that the value of the conductionband offset is larger than the valence-band offset. Clearly more work needs to be done before one can have confidence in the values of the band offsets in this materials system. It should be pointed out that our value of the conduction-band 


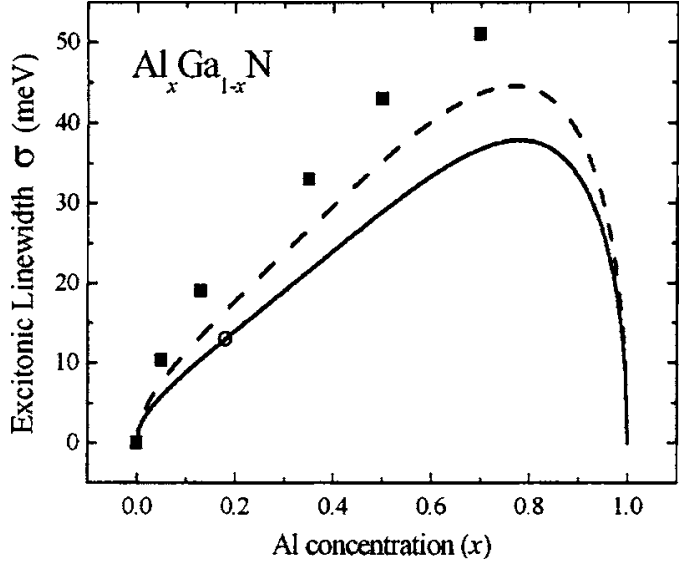

FIG. 2. Variation of the excitonic linewidth $(\sigma)$ as a function of Al concentration $(x)$. The solid and dashed curves are calculated using localized and free exciton models, respectively. The solid squares are the experimental data given in Ref. 3. The open circle denotes the value for the current sample.

offset is the upper limit as the value of $\sigma(13.0 \mathrm{meV})$ may contain contributions from mechanisms other than compositional potential fluctuations.

As mentioned earlier, Coli et al. ${ }^{2,3}$ have measured the variation of $\sigma$ as a function of $\mathrm{Al}$ concentrations in $\mathrm{Al}_{x} \mathrm{Ga}_{1-x} \mathrm{~N}$ at $10 \mathrm{~K}$. They found that their measured values of $\sigma$ agree well with those calculated using the formalism of Lee and Bajaj ${ }^{6}$ that assumes completely random alloys and delocalized excitons. We have now strong evidence that excitons are localized in AlGaN alloys. ${ }^{7}$ We have therefore calculated the variation of $\sigma$ as a function of $\mathrm{Al}$ concentration in $\mathrm{Al}_{x} \mathrm{Ga}_{1-x} \mathrm{~N}$ using our model of the localized excitons as described above. We display our results (solid line) in Fig. 2 along with the results of a delocalized exciton model (dashed line) and the experimental data (solid squares) of Coli et $a l^{3}$ We find that the values of $\sigma$ obtained using the localized exciton model are considerably smaller than those calculated using the delocalized model and the experimental values. This is not surprising. It is well known that the growth of $\mathrm{AlGaN}$ alloys with higher values of $\mathrm{Al}$ concentration always leads to additional incorporation of impurities and defects, larger strain inhomogeneities, and possibly more clustering. The effects of these imperfections on the value of $\sigma$ are not included in our calculations. The difference between our calculated values of $\sigma$ using a localized exciton model and the experimental values is suggested to be due to the effects of these imperfections.

It should be pointed out that our approach to determine the values of the energy-band offsets does not involve the use of interfaces between GaN and AlN. Thus, our values are free from the uncertainties introduced by the presence of strain inhomogeneities, defects, and clustering at the interfaces. It is therefore more meaningful to compare our values of the energy-band offsets with those determined by Foxon et al. ${ }^{15}$ using radiative recombination between different centers in bulk AlGaN. Even though energy-band offsets are defined when two different semiconductors are brought in contact, the values determined by using bulk properties may be closer to their intrinsic values. As mentioned earlier our sample exhibits composition modulation along the direction of growth and is of high structural quality. There may be similar composition modulations in the plane perpendicular to the growth direction as well. It is reasonable to assume that these composition modulations play an important role in completely localizing the hole at low temperatures.

\section{CONCLUSION}

We have measured the value of the linewidth of an excitonic transition in an $\mathrm{Al}_{x} \mathrm{Ga}_{1-x} \mathrm{~N}$ alloy with $x=0.18$ using PL spectroscopy at $10 \mathrm{~K}$. Our sample was grown on (0001)oriented sapphire substrate by MOCVD at $1050{ }^{\circ} \mathrm{C}$. The value of the observed excitonic linewidth $(13.0 \mathrm{meV})$ in this sample is the lowest ever reported in literature. It is significantly smaller than the value calculated using the delocalized exciton model of Lee and Bajaj and is the lowest value reported in the literature. The excitons are known to be strongly localized in this alloy system, and we have simulated this localization by assuming that the hole, being much more massive than the electron, is completely immobilized. In other words we treat its mass as infinite. Considering now that the excitonic linewidth is entirely caused by the potential fluctuations experienced by the electron, the value of the conduction-band discontinuity is determined to be $57 \%$ of the energy-band-gap difference. This value is similar to those recently found in GaN/AIN structures by some other groups.

\section{ACKNOWLEDGMENTS}

One of us (K.K.B.) would like to thank Professor S. Mahajan for his kind and generous hospitality during his stay at Arizona State University. The support of this work by NSF and AFOSR is gratefully acknowledged.

${ }^{1}$ G. Steude, B. K. Meyer, A. Göldner, A. Hoffmann, F. Bertram, J. Christen, H. Amano, and I. Akasaki, Appl. Phys. Lett. 74, 2456 (1999).

${ }^{2}$ G. Coli, K. K. Bajaj, J. Li, J. Y. Lin, and H. X. Jiang, Appl. Phys. Lett. 78, 1829 (2001).

${ }^{3}$ G. Coli, K. K. Bajaj, J. Li, J. Y. Lin, and H. X. Jiang, Appl. Phys. Lett. 80, 2907 (2002).

${ }^{4}$ S. T. Pendlebury et al., Phys. Status Solidi A 188, 871 (2001).

${ }^{5}$ K. K. Bajaj, Mater. Sci. Eng., R. 34, 59 (2001).

${ }^{6}$ S. M. Lee and K. K. Bajaj, J. Appl. Phys. 73, 1788 (1993).

${ }^{7}$ Y. H. Cho, G. H. Gainer, J. B. Lam, J. J. Song, W. Yang, and W. Jhe, Phys. Rev. B 61, 7203 (2000).

${ }^{8}$ I. Vurgaftman, J. R. Meyer, and L. R. Ram-Mohan, J. Appl. Phys. 89, 5815 (2001).

${ }^{9}$ I. Vurgaftman and J. R. Meyer, J. Appl. Phys. 94, 3675 (2003).

${ }^{10}$ J. Zeman, G. Martinez, K. K. Bajaj, I. Krivorotov, and K. Uchida, Appl. Phys. Lett. 77, 4335 (2000).

${ }^{11}$ F. Saidi, F. Hassen, H. Maaref, L. Auvray, H. Dumont, and Y. Monteil, Mater. Sci. Eng., C 21, 245 (2002).

${ }^{12}$ L. F. Bian, D. S. Jiang, P. H. Tan, S. L. Lu, B. Q. Sun, L. H. Li, and J. C. Harmand, Solid State Commun. 132, 707 (2004).

${ }^{13}$ R. T. Senger et al., Appl. Phys. Lett. 81, 2614 (2003).

${ }^{14}$ R. T. Senger et al., Appl. Phys. Lett. 81, 5425 (2003).

${ }^{15}$ C. T. Foxon, S. V. Navikov, L. X. Zhao, and I. Harrison, Appl. Phys. Lett. 83, 1166 (2003).

${ }^{16}$ A. Helman et al., Appl. Phys. Lett. 83, 5196 (2003).

${ }^{17}$ A. Satta, V. Fiorentini, A. Bosin, and F. Meloni, Mater. Res. Soc. Symp. Proc. 395, 515 (1996)

${ }^{18}$ F. Bernardini and V. Fiorentini, Phys. Rev. B 57, R9427 (1998). 